\title{
Text Extraction of Colour Images using Mathematical Morphology \& HAAR Transform
}

\author{
Mansi Agarwal ${ }^{1}$, Adesh Kumar ${ }^{2}$, Vimal Gupta ${ }^{3}$ \\ ${ }^{\prime}$ (M.Tech Scholar, Computer Science \& Engineering, JSS Academy of Technical Education, Noida India \\ ${ }_{2}^{2}$ (Assistant Professor, Department of Electronics, Instrumentation\& Control Engineering, University of \\ Petroleum \& Energy studies, Dehradun India, \\ ${ }^{3}$ (Assitant Professor, Department of Computer Science \& Engineering, JSS Academy of Technical Education, \\ Noida, India,
}

\begin{abstract}
Digital image processing is an ever expanding and dynamic area with applications reaching out into our daily life such as digital signature, authentication, surveillance, medicine, space exploration, automated industry inspection and many others areas. Theseapplications are involved in different processes like image enhancement, object detection, features extraction, colour imaging etc. Implementation of such applications on a general purpose computer can be easier, but every time it is not efficient due to additional constraints on memory and other peripheral devices. Out of the five senses - sight, hearing, touch, smell and taste, humans use to perceive their environment. Among all,sight of images is the most powerful. More than $99 \%$ of the activity of the human brain is involved in processing images from the visual cortex. A visual image is rich in information. There is an efficient yet simple method to extract text regions from video sequences or static images. The speed of Haar discrete wavelet transform (DWT) operates the fastest among all wavelets because its coefficients are either 1 or -1. It is one of the reasons that Haar DWT is used to detect edges of candidate text regions. Image sub bands contain both text edges and non-text edges. The intensity of the text edges is also different from that of the non-text edges. Therefore, thresholdingis used to preliminary remove the non-text edges. Text regions of colour images are composed of horizontal edges, vertical edges and diagonal edges. Morphological dilation operators as AND, OR are applied to connect isolated text edges of each detail component sub-band in a transformed binary image. The simulation is carried out on MATLAB 2012 image processing tool.
\end{abstract}

Keywords: Discrete Wavelet Transform (DWT), HAAR Transform, Mathematical Morphology

\section{Introduction}

Integrated image capture, processing, and communication power on a compact, portable, hand-held device is attracting and has increased interest from computer vision researchers with a goal of applying a diverse collection of vision tasks on the small hand held device. Thetrend of vision applications is focused on camera phones and proposes solutions to the major technical challenges involving mobile vision and pattern recognition.Recent studies in the field of computer vision and pattern recognition show a great amount of interest in content retrieval from images and videos. This content can be in the form of objects, texture, color, shape as well as the relationshipsbetween them. The semantic information provided by an image can be useful for content based image retrieval, as well as for indexing and classification purposes. As statedby Jung, Kim and Jain in [4], text data is particularly interesting, because text can be used to easily and clearly describe the contents of an image. Text data can be embedded into an image or video in different font styles, sizes, colors, orientations and against a complex background, the problem of extracting the candidate text region becomes a challenging one. Also, current Optical Character Recognition (OCR) techniques can only handle text against a plain monochrome background and cannot extract text from a textured or complex background.

Different approaches for the extraction of text regions from images have been proposed based on basic properties of text. Text has some common distinctive characteristics in terms of frequency, spatial cohesion and orientation information. Spatial cohesion refers to the fact that text characters of the same string appear close to each other and are of similar height, orientation and spacing. Two of the main methods commonly used to determine spatial cohesion are based on edge [1] [2] and connected component [3] features of text characters. The fact that an image can be divided into categories depending on whether or not it contains any text data can also be used to classify candidate text regions. Thus other methods for text region detection, utilize classification techniques such as support vector machines, k-means clustering [7] and neural network based classifiers [10]. The algorithmproposed in [8] uses the focus of attention mechanism from visual perception to detect text regions. Text in images and video sequences pro-vide highly condensed information about thecontents of the images or videos sequencesand can be used for video browsing/retrievalin a large video database. Although texts provide important information about images or video sequences. Detection and segmentation of video or image sequences is not an easy problem. Text extraction is not easy for 
the following reasons. First of all, text sizes may vary from smaller to big and text fonts may vary in a wide range as well.Secondly, texts present in an image or a videosequence may have multiple colors and appear in very much cluttered background.

Many papers about the extraction of texts from static image or video sequence have been published in recent years. Those methods for texts extraction can be classified as either component based or texture based. In component based text extraction methods, different text regions are detected by analyzing the edges of the candidate regions or homogenous color/grayscale components that contain the characters. For example, Park et al. [1] detected eight orientations of edge pixels in the documents using prewitt masks. In imaging, edge pixels can be classified as either diagonal directional or axial directional. They dilate two kinds of edge pixels using morphological dilation operators. Logical AND is applied to these two edges to obtain the real text regions. Zhong et al. [2] located bounding boxesaround text components using the horizontal spatial variance. Non text regions have lower horizontal spatial variance than that of candidate text regions. The connected components in each candidate text region are of the same color intensity. They determined the color of texts and locate text components in each candidate text region. Finally, real text components are filled in each candidate text region. Chen et al. [3] detected vertical edges and horizontal edges in an image and dilated two kinds of edges using different dilation operators. The logical AND operator is performed on dilated vertical edges and dilated horizontal edges to obtain candidate text regions. Real Text regions are then identified using support vector machine. Text regions usually have a special texture because they consist of identical character components. These components also contrast the background and hence text regions have a periodic horizontal intensity variation due to the horizontal alignment of characters. As a result, text regions can be segmented using texture features. For example, Stephen et al. [4] did the segmentation and labeling of block using the connected component analysis. Paul et al [5] segmented and classified texts in a newspaper by generic texture analysis. Small masks are applied to obtain local textural characteristics.

Most of the text extraction methods were applied to uncompressed images. Few of them proposed to extract texts in the compressed version of images. Zhong et al. [6] extracted captions from the compressed videos (MPEG video and JPEG image) based on Discrete Cosine Transform (DCT). DCT detects edges in different directions from the candidate image. Edge regions containing texts are then detected using a threshold afterward. Acharyya et al. [7] segmented texts in the document images based on wavelet scale-space features. The method used the M-band wavelet which decomposes an image into some $\mathrm{M} \times \mathrm{M}$ band pass channels so as to detect the text regions easily. The intensity of the candidate text edges are used to recognize the real text regions in an M-band image.

Research objective is to extract the text form the colored images using image processing tool. The extraction of text is carried out from any image using Haar Transform. Character reorganization from the image and character extraction form the colored image is also the objective of image extraction. A method to extract texts in images or video sequences using Haar discrete wavelet transform (Haar DWT) is also presented. The edges detection is accomplished by using 2-D Haar DWT and some of the non-text edges are removed using thresholding. After it, different morphological dilation operators to connect the isolated candidate text edges in each detail component sub-band of the binary image. Morphological dilation operators also extract the characters from the images using mathematical morphological and templates.

\section{Mathematical Morphology}

Mathematical morphology (MM) [1] is a nonlinear branch of the signal processing field and concerns the application of set theory concepts to image analysis and processing. Morphology refers to the study of shapes and structures from a general scientific perspective. Geometric features of images are modified using morphological filters or operators as nonlinear transformations. These operators transform the original image into another image through the iteration with other image of a certain shape and size which is known as structuring element.In general, the structuring element is a set that describes a simple shape that probes an input image. Morphological filters are based on morphological opening and morphological closing with structuring elements. The present filters have several inconveniences in some situations. The image structure is changed general, if undesirable features are eliminated.On the other hand, an image by reconstruction [5][7]has become a powerful tool that enables us to eliminate undesirable features without necessarily affecting desirable properties. From a practical perspective images by reconstruction are built by means of a reference image, background and foreground markers. In the paper morphological image reconstruction based algorithm are used to obtain the results better than general opening (resp., closing)by reconstruction and to avoid some of the inconveniences.Image segmentation is one of the most important categories of image processing. The main purpose of image segmentation is to divide an original image into homogeneous regions. Image segmentation can be applied as a preprocessing stage for other image processing methods. Several approaches are available for image segmentation methods for image processing. 


\subsection{Features of Morphology}

The term morphology refers to the study of shapes and structures from a general scientific perspective. It is also interpreted as shape study using mathematical set theory. In image processing, morphology is the name of a specific methodology for analyzing the geometric structure inherent within an image. The morphological filter, which can be constructed on the basis of the underlying morphological operations, can be more suitable for shape analysis than the standard linear filters since the latter sometimes distort the underlying geometric form of the image.In general, morphological operators transform the original image into another image through the interaction with the other image of a certain shape and size, which is known as the structuring element. The geometric features of an image that are similar in shape and size to the structuring element are preserved.But other features of image are suppressed default. Therefore, morphological operations can simplify the image data, preserving their characteristics, shape and eliminate irrelevancies. In view of applications, morphological operations can be employed for many purposes including segmentation, edge detection, and enhancement of images. Mathematical morphology is based on geometry. Set theory is the theoretical foundations of morphological image processing and the mathematical theory of order. General idea to solve the problem for an image is the use of template shape, which are called structuring elements to quantify the manner in which the structuring element fits within a given image.

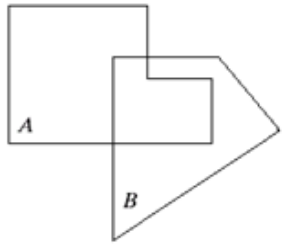

(a) Two image $A$ and $B$

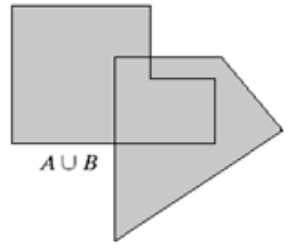

(b) Union

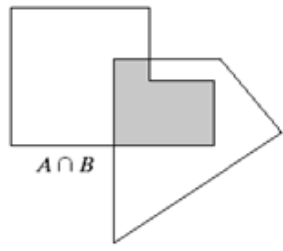

(c) Intersection

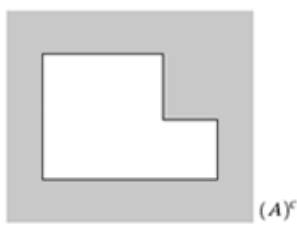

(d) Complement

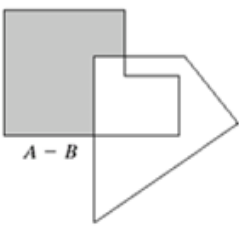

(e) Difference

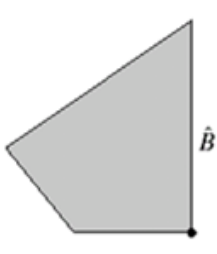

(f) Reflection of $B$

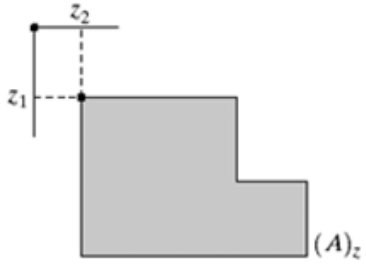

(g) Translation

Fig. 1 Set Theory operations [11]

\section{Union}

The union of two images $\mathrm{A}$ and $\mathrm{B}$ is written as $A \cup B$. The meaning of $A \cup B$ is that it is a set whose elements are either the elements of $\mathrm{A}$ or $\mathrm{B}$ or of both. The definition is given by

$$
A \cup B=\operatorname{defi}(x) x \in A \text { or } x \in B
$$

\section{Intersection}

The union of two images $\mathrm{A}$ and $\mathrm{B}$ is written as $A \cap B$. The meaning of $A \cap B$ is that it is a set whose elements are common to both images $\mathrm{A}$ or $\mathrm{B}$. The definition is given by

\section{Difference}

$$
A \cap B=\operatorname{def}\{x \mid x \in A \text { and } x \in B\}
$$

The difference of two images $\mathrm{A}$ and $\mathrm{B}$ is written as $A-B$. The difference $A-B$ subtracts from image A which are in image B. It is also called the relative complement of B relative to A. The definition is given by

\section{Complement}

$$
\left.A-B=\operatorname{defi}\left|\operatorname{cix}_{x}\right| x \in A \text { and } x \notin B\right\}
$$

The complement of image $\mathrm{A}$ is written $\mathrm{A}^{\mathrm{c}}$. In complement set it is consisting which is not available in image A. The definition is given by

\section{Reflection}

$$
\left.A^{c}=\operatorname{defifx} \mid x \notin A\right\}
$$

The refection of an image $\mathrm{B}$ can be defined as

$$
B_{1}=\{w \mid w=-b \quad \text { for } b \in B\}
$$




\section{Translation}

In translation the origin of an image $\mathrm{A}$ is shifted to some points, can be defined as

$$
A_{1}=\{c \mid c=a+z \text { for } a \in A\}
$$

Figure 1 shows the all set operations sequentially.

\subsection{Logical Operations}

OR operation is similar to the union operation. AND operation is similar to intersection operation. Not operation is similar to complement operation. If the pixels of image A and B are complement to each other, then the resultant image pixel is black, else the resultant pixel is white.

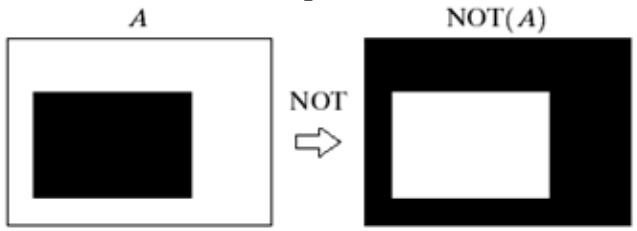

(a) Not operation of $\mathbf{A}$
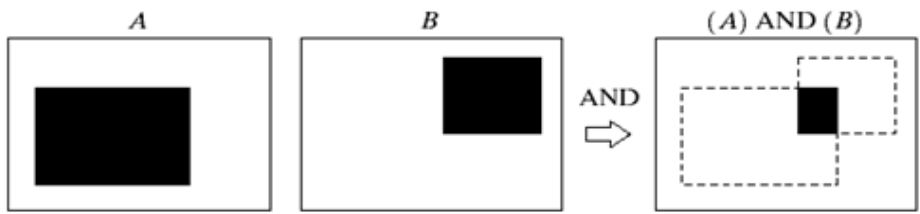

(b) AND operation
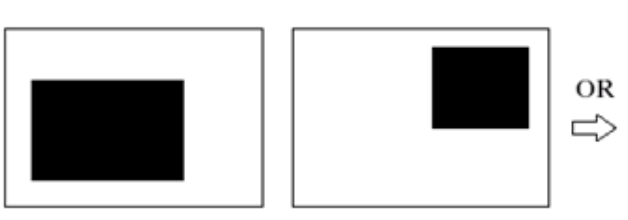

(A) OR $(B)$

(c) OR operation
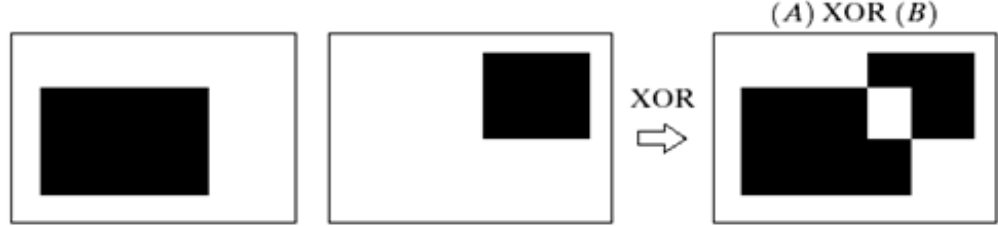

(d) XOR logic

\subsubsection{Binary Dilation}

Fig. 2 Logical operations [11]

Definition: Binary Dilation

With $\mathrm{A}$ and $\mathrm{B}$ assets in $\mathrm{Z}$, the dilation of A by B (usually A is an image and B is the structuring element), denoted by $\mathrm{A} \oplus \mathrm{B}$, is defined as

$$
\mathrm{A} \oplus \mathrm{B}=\left\{z \in Z^{2} \mid z=a+b \text {, for } a \in A \text { and } b \in B\right\}
$$

Dilationis defined as a union of translation of the original image with respect to the structuring element.

$$
\mathrm{A} \oplus B=\mathrm{U}_{b \in B}(A) b
$$

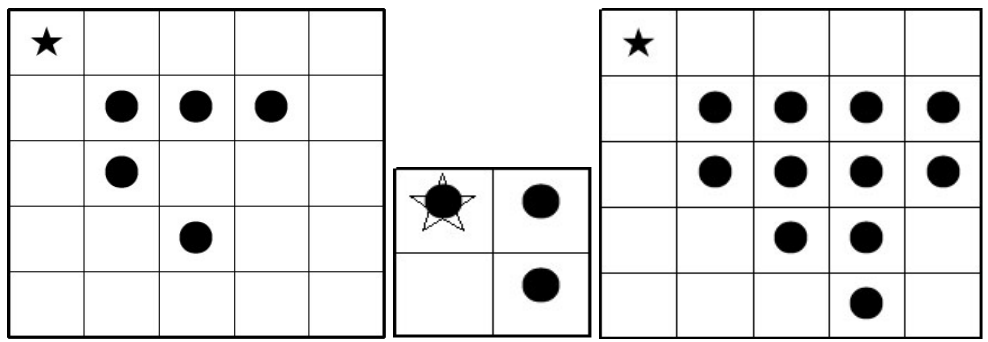

Fig. 3 Illustration of Binary Dilation on Digital Setting Dilation is found by placing the center of the template over each of the foreground pixels of the original image and then taking the union of all the resulting copies of the structuring element using translation. 
From figure 3 , it is clear how dilation modifies the original image with respect to the shape of the structuring element. Dilation generally has an effect of expanding an image; so consequently, small holes inside foreground can be filled.In another sense, dilation can be a morphological operation on a binary image defined as

$$
\mathrm{A} \oplus \mathrm{B}=\{\mathrm{Z} \mid(\widehat{B}) \mathrm{z} \cap \mathrm{A} \neq \emptyset\}
$$

This equation is based on obtaining the reflection of $\mathrm{B}$ about its origin and shifting this reflection by $\mathrm{Z}$. In dilation the set of all displacements such that B and A overlap with A by B isusing at least one element.Based on this interpretation, the equation above may be written as

$$
\mathrm{A} \oplus \mathrm{B}=\{\mathrm{Z} \mid[(\hat{B}) \mathrm{z} \cap \mathrm{A}] \underline{\mathrm{C}} A\}
$$

Although dilation is based on set theory, but convolution is based on arithmetic operations, the basic process "flipping" B about its origin and successively "displacing" it so that it slides over set Ais analogous to the convolution process. Even though dilation of an image A by structuring element B can be defined in several ways, all definitions have the same meaning and results in the same output.

\subsubsection{Binary Erosion}

Definitions: Binary Erosion

Erosion of a binary image $\mathrm{A}$ by structuring element $\mathrm{B}$, denoted by $\mathrm{A} \Theta \mathrm{B}$, is defined as $\mathrm{A} \Theta \mathrm{B}=\{\mathrm{Z} \mid \mathrm{Z}+\mathrm{b} \in \mathrm{A}, \forall \mathrm{b} \in \mathrm{B}\}$

Whereas image dilation can be represented as a union of translates, erosion can be represented as an intersection of the negative translates. Erosion can be redefined as

$$
\mathrm{A} \Theta \mathrm{B}=\cup_{b \in B} A-b
$$

Where ' $-\mathrm{b}$ ' is the scalar multiple of the vector $b$ by -1 . Figure 4 shows the binary erosion.

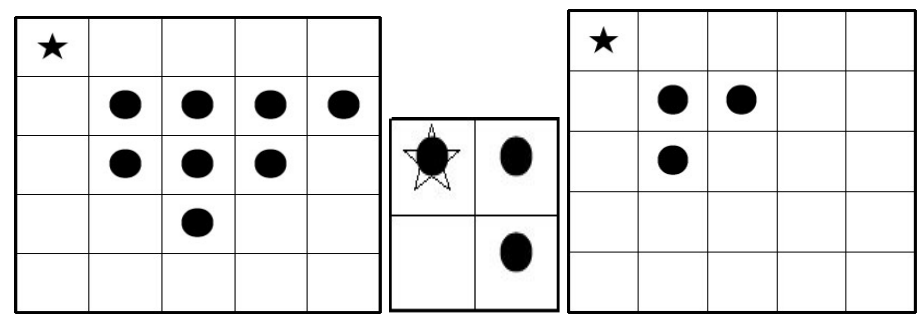

Fig.4 Illustration of binary erosion on digital setting

The erosion of the original image by the structuring element can be described intuitively by template translation as seen in the dilation process. Erosion shrinks the original image and eliminates small enough peaks.

\subsubsection{Binary opening}

\section{Definition: Binary Opening}

Opening is a morphological operation based on erosion and dilation. The opening of a binary image $\mathrm{A}$ by the structuring element $\mathrm{B}$, denoted by $(A \circ B)$ is defined

$$
A \circ B=(A \ominus B) \oplus B
$$

First erode A by B, and then dilate the result by $\mathrm{B}$. In other words, opening is the unification of all $\mathrm{B}$ objects entirely contained in A. Figure 5 shows the opening of two images A and B.

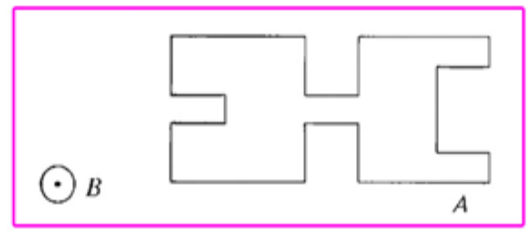

(a)

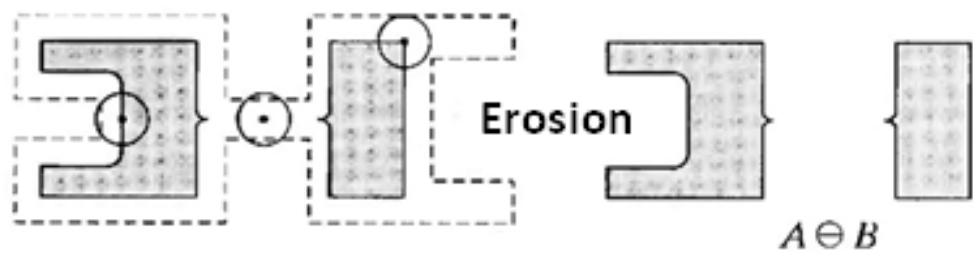

(b) 

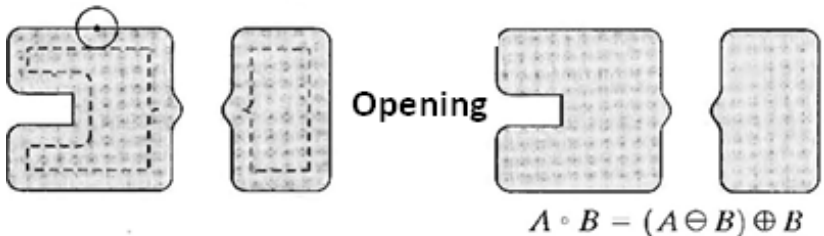

\subsubsection{Closing}

Fig. 5 (a) input image $A$ and $B$ (b) erosion(c) Opening [11]

The closing is the opposite of opening. In opening dilation operation is followed by an erosion process. First, dilate A by B, and then erode the result by B. In other words, closing is the group of points, which the intersection of object B around them with object A is not empty. Figure 6 shows the closing of two images A and $\mathrm{B}$.

$$
A \cdot B=(A \oplus B) \oplus B
$$

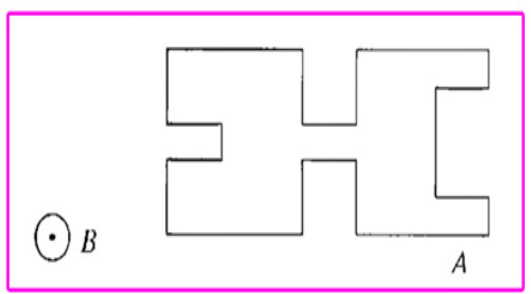

(a)
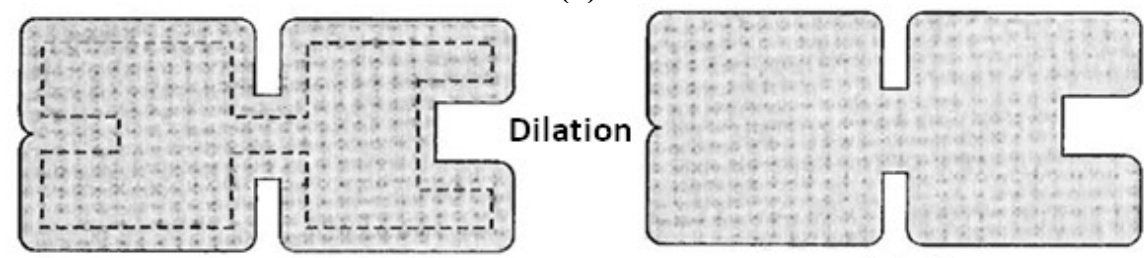

$A \oplus B$

(b)
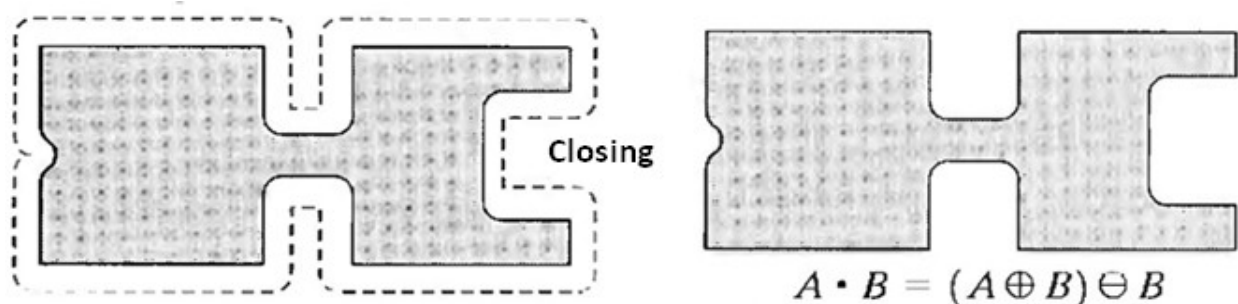

$A \cdot B=(A \oplus B) \ominus B$

(C)

Fig. 6(a) image A and B (b) Dilation process (c) Closing [11]

\section{Haar Transform \& Image Extraction}

In Haar Transform, is a method to extract texts in images using Haar discrete wavelet transform (Haar DWT). The edges detection is accomplished by using 2-D Haar DWT and some of the non-text edges are removed using thresholding. In the DWT transform, an image signal can be analyzed by passing it through an analysis filter bank followed by decimation operation. This filter is the combination of a low pass filter and high pass filter at each decomposition level. 


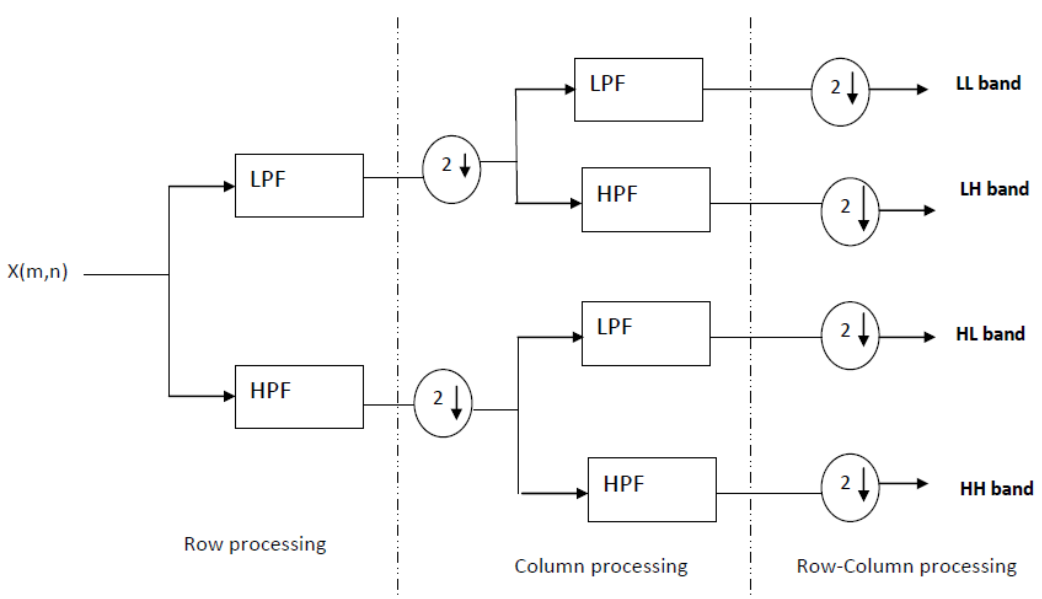

Fig.7 Haar Transform decomposition

. When the signal passes through these filters, it splits into bands. The coarse information is extracted by the low pass filter which corresponds to an averaging operation. The detail information of the signal is extracted by high pass filter because it is corresponds to differencing operation. Two dimensional transform is done by the separation of 1D transforms and output of image is decimated by 2, first with respect to row and then following the filtering of sub images. Now sub images are separated by 2 with respect to column. Afterward, we use different morphological dilation operators to connect the isolated candidate text edges in each detail component sub-band of the binary image. Although the color component may differ in a text region but the information about colors does not help extracting texts from images. For gray-level image, input image is processed directly starting at discrete wavelet transform. This operation separates the image into four possible bands LL, LH, HL and $\mathrm{HH}$ respectively.

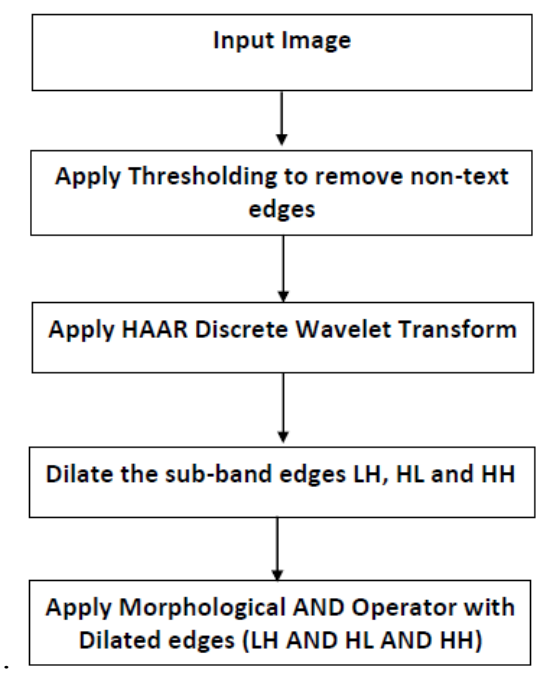

Fig. 8 Flow chart of the proposed text follows

If the input image is colored, its RGB components are combined to give an intensity image $\mathrm{Y}$ as

$$
\mathrm{Y}=0.299 \mathrm{R}+0.587 \mathrm{G}+0.114 \mathrm{~B}
$$

Image $\mathrm{Y}$ is then processed with discrete wavelet transform and the whole extraction algorithm afterward. The flow chart of the proposed algorithm is shown in figure 8. If the input image itself is stored in the DWT compressed form, DWT operation can be omitted in the proposed algorithm.

\subsection{Haar discrete wavelet transform}

The discrete wavelet transform is a very useful tool for signal processing and image analysis especially in multi-resolution representation. In DWT signals are decomposed into different components in the frequency domain. 1-D DWT decomposes an input sequence into two components the average component and the detail component by calculations with a low-pass filter and a high-pass filter [9]. Two-dimensional discrete wavelet 
transform (2-D DWT) decomposes an input image into four sub-bands, one average component (LL) and three detail components (LH, HL, HH) as shown in figure 9. In image processing, the multi-resolution of 2-D DWT has been employed to detect edges of an original image. However, 2-D DWT can detect three kinds of edges at a time while traditional edge detection filters cannot. As shown in figure 10the traditional edge detection filters detect three kinds of edges by using four kinds of mask operators. Therefore, processing times of the traditional edge detection filters is slower than 2-D DWT.

\begin{tabular}{|l|l|}
\hline LL & HL \\
\hline LH & HH \\
\hline
\end{tabular}

Fig. 9 2-D DWT decomposition scheme

Figure 10 shows a gray level image. The 9-7 taps DWT filters decompose this gray image into four subbands as shown in Figure 10 (b). As we can see, three kinds of edges present in the detail component sub-bands but look unobvious. The detected edges in image become more obvious and the processing time decreases, if we replace the 9-7 taps DWT filters with Haar DWT. The operation for Haar DWT is simpler than that of any other wavelets and applied to image processing especially in multi-resolution representation. Haar DWT has the following important features [17].

- Haar wavelets are real, symmetric and orthogonal.

- Its boundary conditions are the simplest among all wavelet-based methods.

- The minimum support property allows arbitrary spatial grid intervals.

- It can be used to analyze texture and detect edges of characters.

- The high-pass filter and the low-pass filter coefficientsare simple (either 1 or -1 ).

Figure 11 shows an example of $256 \times 256$ images, in which it is divided into $128 \times 128 \mathrm{LL}, \mathrm{LH}, \mathrm{HL}$ and LL bands. Further the image is divided into $64 \times 64$ LLLL, LLLH, LLHL and LLHH bands as shown in figure 12 (a) and (b).

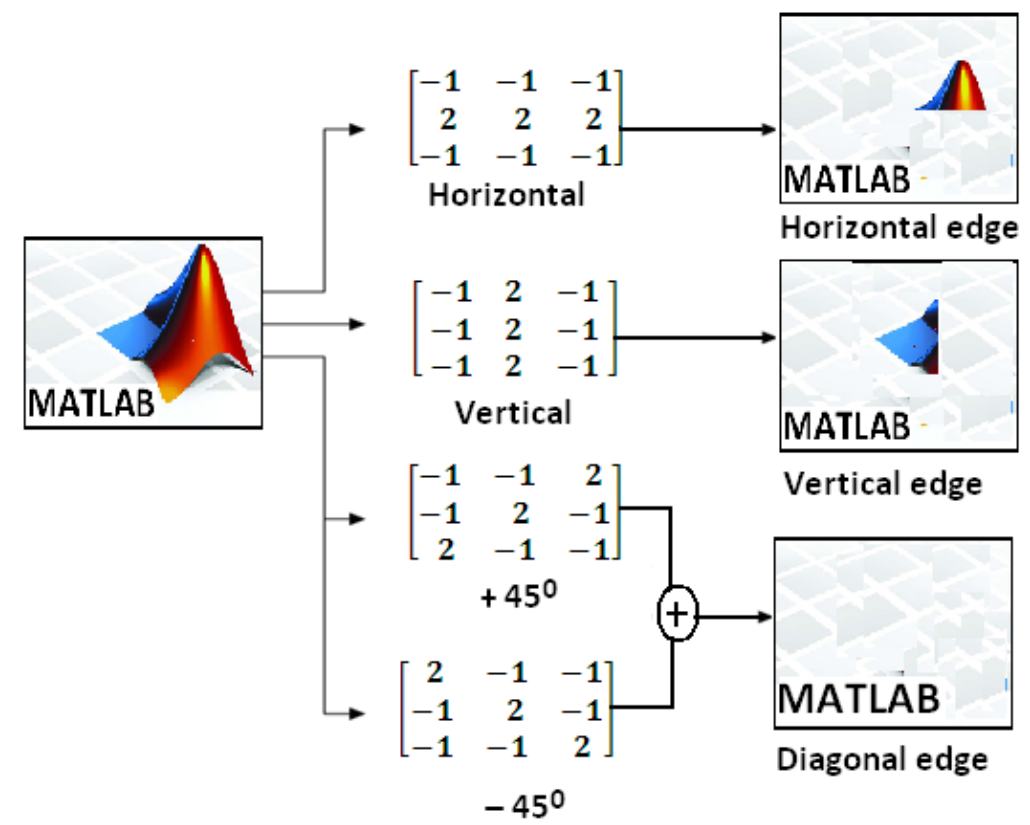

Fig. 10 Traditional edge detection using mask operation

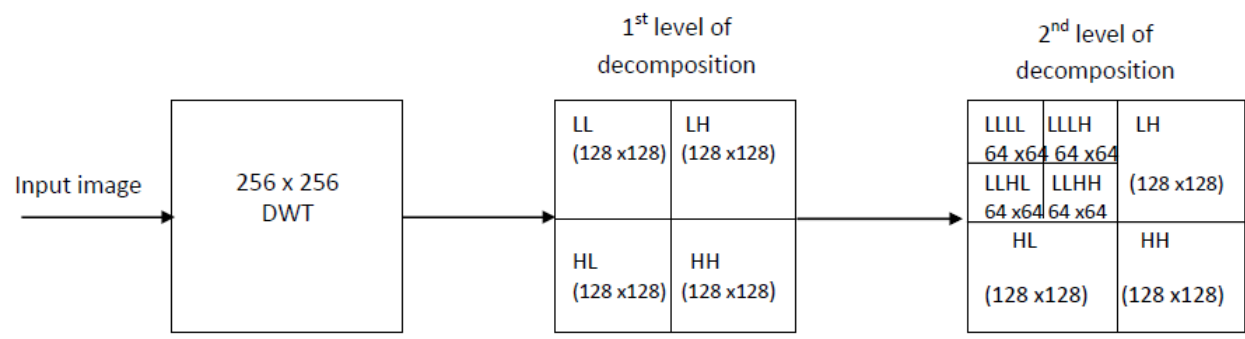

Fig.11 Decomposition scheme of Haar Transform 

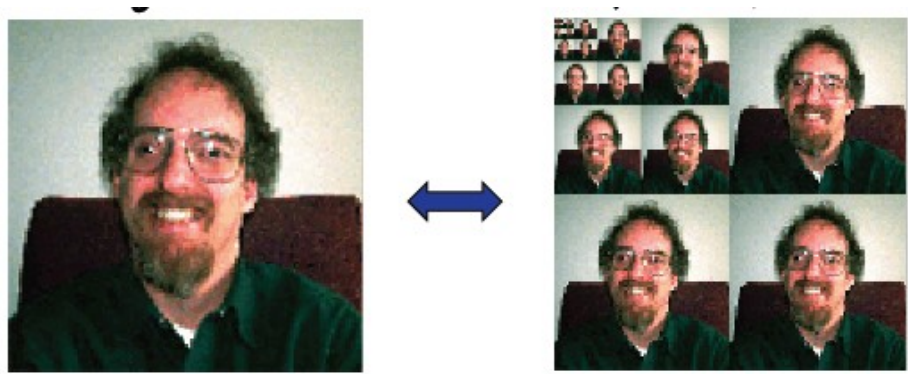

Fig. 12(a) Original gray image (b) DWT coefficients

Figure 13 (a) matrix shows the example of a $4 \times 4$ colour image. The wavelet coefficients can be obtained in gray-level image using addition and subtraction. 2-D DWT is achieved by two ordered 1-D DWT operations based on row and column values. First of all, row operation is performed to obtain the result shown in figure 13 (b)matrix. Then it is transformed by the column operation and the final resulted 2-D Haar DWT is shown in Figure 13(c) matrix. 2-D Haar DWT decomposes a gray-level image into one average component subband and three detail component sub-bands.

$$
\begin{gathered}
{\left[\begin{array}{llll}
a & b & c & d \\
e & f & g & h \\
i & j & k & l \\
m & n & o & p
\end{array}\right] \quad\left[\begin{array}{cccc}
(a+b) & (c+d) & (a-b) & (c-d) \\
(e+f) & (g+h) & (e-f) & (g-h) \\
(i+j) & (k+l) & (i-j) & (k-l) \\
(m+n) & (o+p) & (m-n) & (o-p)
\end{array}\right]} \\
{\left[\begin{array}{llll}
(a+b)+(e+f) & (c+d)+(g+h) & (a-b)+(e-f) & (c-d)+(g-h) \\
(i+j)+(m+n) & (k+l)+(o+p) & (i-j)+(m-n) & (k-l)+(o-p) \\
(a+b)-(e+f) & (c+d)-(g+h) & (a-b)-(e-f) & (c-d)-(g-h) \\
(i+j)-(m+n) & (k+l)-(o+p) & (i-j)-(m-n) & (k-l)-(o-p)
\end{array}\right]}
\end{gathered}
$$

Figure 13 Matrixes (a) The original image (b) the row operation of 2-D Haar DWT and (c) column

\section{Result \& Discussion}

The simulation work of MATLAB text extraction is tested on different jpg and bmp colour images and the text extraction is done successfully. For different test cases the results are shown the figure 14.

Haar Transform is a good method to transform the text from the colour images. A method of text extraction from images is proposed using the Haar Discrete Wavelet Transform, the Sobel edge detector, the weighted OR operator, thresholding and the morphological dilation operator. Mathematical morphological techniques are used for the detection of text and characters. We have created the templates for the all characters and these templates are used for the edge detection using mathematical morphological operators. These mathematical tools are integrated to detect the text regions from the complicated images. The proposed method is robust against language and font size of the texts. The proposed method is also used to decompose the blocks including multi-line texts into single line text. According to the experimental results, the proposed method is proved to be efficient for extracting the text regions from the images. In future we can use the techniques to extract the special characters and large volume of data.

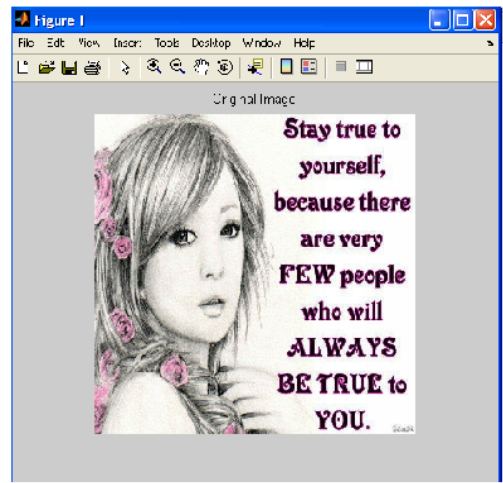

(a)

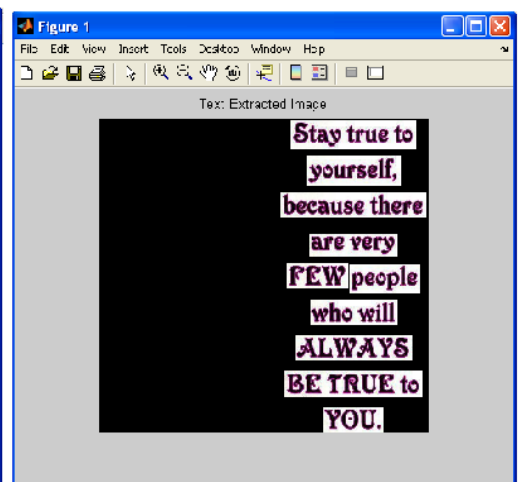

(b) 


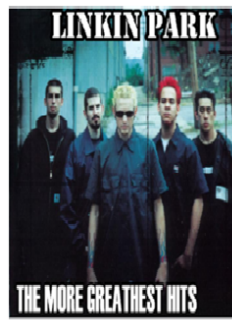

(c)

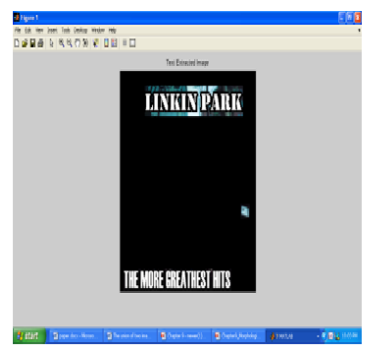

(d)

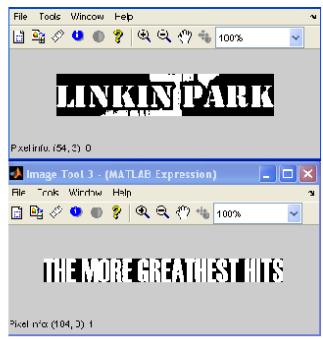

(e)

\section{Mtu123 MANSI INDIA}

(f)

(g)

Fig. 14 Tested images for text and character extraction

\section{Conclusion}

The text extraction on the colour images using mathematical morphology and Haar DWT is done successfully. Applications of text extraction are huge including the making of digital copies of the ancient scripture to everyday life bills etc. It may be required to be of digital form. This setup can be used to recover textual information from surveillance footage, satellite imaging, tollbooth as in a hybrid approach is used to extract textual information form a video scene. Major application of it is that it can be used in license plate recognition of a vehicle as in an approach to determine license plate number. In the future work we can explore the techniques to recognize the special characters from colour images.

\section{References}

[1] Xiaoqing Liu and JagathSamarabandu, An Edge-based text region extraction algorithm for Indoor mobile robot navigation, Proceedings of the IEEE, July 2005.

[2] Xiaoqing Liu and JagathSamarabandu, Multiscale edge-based Text extraction from Complex images, IEEE, 2006.

[3] JulindaGllavata, Ralph Ewerth and Bernd Freisleben, A Robust algorithm for Text detection in images, Proceedings of the $3^{\text {rd }}$ international symposium on Image and Signal Processing and Analysis, 2003.

[4] Keechul Jung, KwangIn Kim and Anil K. Jain, Text information extraction in images and video: a survey, The journal of the Pattern Recognition society, 2004.

[5] Kongqiao Wang and Jari A. Kangas, Character location in scene images from digital camera, The journal of the Pattern Recognition society, March 2003.

[6] K.C. Kim, H.R. Byun, Y.J. Song, Y.W. Choi, S.Y. Chi, K.K. Kim and Y.K Chung, Scene Text Extraction in Natural Scene Images using Hierarchical Feature Combining and verification, Proceedings of the 17thInternational Conference on Pattern Recognition (ICPR '04), IEEE.

[7] Victor Wu, RaghavanManmatha, and Edward M. Riseman, TextFinder: An Automatic System to Detect and Recognize Text in Images, IEEE Transactions on Pattern Analysis and Machine Intelligence, Vol. 21, No. 11, November 1999.

[8] Xiaoqing Liu and JagathSamarabandu, A Simple and Fast Text Localization Algorithm for Indoor Mobile Robot Navigation, Proceedings of SPIE-IS\&T Electronic Imaging, SPIE Vol. 5672, 2005.

[9] Qixiang Ye, Qingming Huang, Wen Gao and Debin Zhao, Fast and Robust text detection in images and video frames, Image and Vision Computing 23, 2005.

[10] Rainer Lienhart and Axel Wernicke, Localizing and Segmenting Text in Images and Videos, IEEE Transactions on Circuits and Systems for Video Technology, Vol.12, No.4, April 2002.

[11] R.C. Gonzales and R.E. Woods, Digital Image Processing, Addison-Wesley, Reading, 1992.

[12] J.D. Foley, A. van Dam, S.K. Feiner and J.F. Hughes, Computer Graphics, Principles and Practice, Addison-Wesley, Reading, 1990.

[13] Park, C. J., Moon, K. A., Oh, Weon- Geun, and Choi, H. M. 2000. An efficient of character string positions using morphological operator. IEEE International Conference on Systems, Man, and Cybernetics, 3, 8-11: 1616-1620.

[14] Zhong, Yu., Karu, K., and Jain, A.K. 1995. Locating text in complex color images. Proceedings of the Third International Conference on Document Analysis and Recognition, 1, 14-16: 146-149.

[15] Chen, Datong, Bourlard, H., and Thiran J. P., 2001. Text identification in complex background using SVM. IEEE Computer Society Conference on Computer Vision and Pattern Recognition, Proceedings of the 2001, 2, 8-14:621-626.

[16] Lam, S. W., Wang, D., and Srihari, S. N., 1990. Reading newspaper text.International Conference on Pattern Recognition Proceedings, 10th. I, 16-21: 703-705.

[17] W. Sowmya, Roy PailyIn, "FPGA implementation of Image Enhancement Algorithm" International conference on Communication and signal processing(ICCSP) IEEE pp 584-588, 2011

[18] K. Sri Rama Krishna, A. Guruva Reddy, M. N. Giri Prasad “A Fault Tolerant FPGA based image Enhancement Filter using Self Healing Algorithm” International Journal of Engineering Science and Technology Vol 2, pp 4922-4928 2010.

[19] ArnabSinha and SumanaGupta,"A Fast Nonparametric Non causal MRF-Based Texture Synthesis Scheme Using a Novel FKDE Algorithm" IEEE Transactions on Image Processing, No.3, March 2010.

[20] Yuichi Tanaka,Madoka Hasegawa, Shigeo Kato, Masaaki Ikehara, and Truong Q. Nguyen, "Adaptive Directional Wavelet Transform Based on Directional Pre filtering”, IEEE Transactions on Image Processing, Volume 19, No.4, April 2010. 